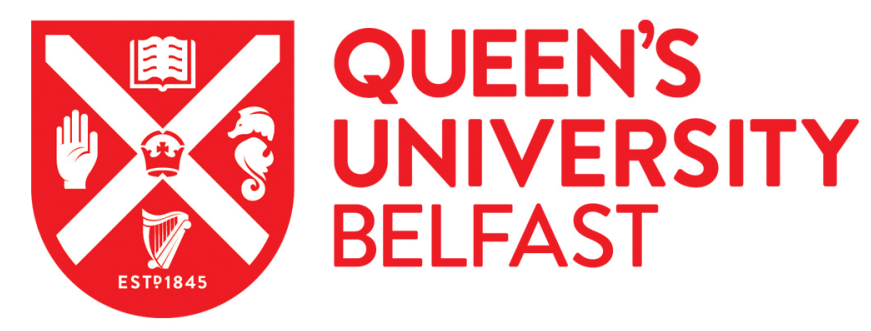

\title{
Experimental Observation of Attosecond Control over Relativistic Electron Bunches with Two-Colour Fields
}

Yeung, M., Rykovanov, S., Bierbach, J., Li, L., Eckner, E., Kuschel, S., Woldegeorgis, A., Rödel, C., Sävert, A., Paulus, G. G., Coughlan, M., Dromey, B., \& Zepf, M. (2016). Experimental Observation of Attosecond Control over Relativistic Electron Bunches with Two-Colour Fields. Nature Photonics, 11, 32-35.

https://doi.org/10.1038/nphoton.2016.239

Published in:

Nature Photonics

Document Version:

Peer reviewed version

Queen's University Belfast - Research Portal:

Link to publication record in Queen's University Belfast Research Portal

Publisher rights

(c) 2016 Macmillan Publishers Limited, part of Springer Nature. All rights reserved. This work is made available online in accordance with the publisher's policies.

\section{General rights}

Copyright for the publications made accessible via the Queen's University Belfast Research Portal is retained by the author(s) and / or other copyright owners and it is a condition of accessing these publications that users recognise and abide by the legal requirements associated with these rights.

Take down policy

The Research Portal is Queen's institutional repository that provides access to Queen's research output. Every effort has been made to ensure that content in the Research Portal does not infringe any person's rights, or applicable UK laws. If you discover content in the Research Portal that you believe breaches copyright or violates any law, please contact openaccess@qub.ac.uk. 
Experimental Observation of Attosecond Control over Relativistic Electron Bunches with Two-Colour Fields

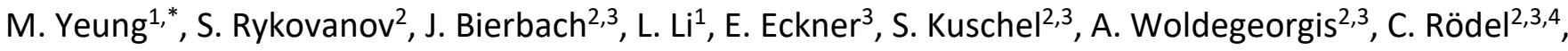
A. Sävert ${ }^{3}$, G. G. Paulus ${ }^{2,3}$, M. Coughlan ${ }^{1}$, B. Dromey ${ }^{1}$ and M. Zepf $f^{1,2,3}$

${ }^{1}$ Department of Physics and Astronomy, Queen's University Belfast, Belfast, BT7 1NN, UK

${ }^{2}$ Helmholtz Institute Jena, Fröbelstieg 3, 07743 Jena, Germany

${ }^{3}$ Institut für Optik und Quantenelektronik, Friedrich-Schiller-Universität Jena, Max-Wien-Platz 1, 07743 Jena, Germany

${ }^{4}$ SLAC National Accelerator Laboratory, 2575 Sand Hill Road, Menlo Park, California 94025, USA

*email: m.yeung@qub.ac.uk

Energy coupling during relativistically intense laser-matter interactions is encoded in the attosecond motion of strongly driven electrons at the pre-formed plasma-vacuum boundary. Studying and controlling this motion can reveal details about the microscopic processes that govern a vast array of light-matter interaction phenomena, including those at the forefront of extreme laser-plasma science such as laser-driven ion acceleration ${ }^{1}$, bright attosecond pulse generation ${ }^{2,3}$ and efficient energy coupling for the generation and study of warm dense matter ${ }^{4}$. Here we experimentally demonstrate that by precisely adjusting the relative phase of an additional laser beam operating at the second harmonic of the driving laser, the trajectories of relativistic electron bunches formed during the interaction with a solid target can be controlled on an attosecond scale. We observed significant enhancements in the resulting high harmonic yield, suggesting potential applications for sources of ultra-bright, extreme-ultraviolet (XUV) attosecond radiation to be used in atomic and molecular pumpprobe experiments 5 . 
During the rising edge of a sufficiently intense laser pulse (peak intensity $>10^{18} \mathrm{Wcm}^{-2}$ ), incident on a solid density target, a plasma is formed such that the peak of the pulse interacts with a dense plasma surface. Electrons formed at such a surface can be driven to relativistic velocities within a single optical cycle and subsequently emit XUV radiation. For multi-cycle laser pulses, these XUV bursts are generated periodically at the driving laser frequency and the radiation is emitted in the form of a train of attosecond pulses observed in the spectral domain as high order harmonics. This emission can be modelled as a periodic Doppler upshift as the electrons move towards the incident laser at velocities close to the speed of light, c, following a description known as the Relativistically Oscillating Mirror (ROM) model ${ }^{7-9}$. Notably, under certain conditions the electrons form extremely short, dense nanobunches that can undergo synchrotron-like trajectories; in the latter case, the XUV generation mechanism is more accurately modelled as Coherent Synchrotron Emission (CSE) ${ }^{2}$.

Recent numerical simulations suggested that using a two-colour field, consisting of the fundamental $(1 \omega)$ pulse and its second harmonic $(2 \omega)$ with a well-defined and controlled relative phase, can optimise the trajectories of the emitting surface electrons leading to a significant increase of the attosecond XUV yield ${ }^{10}$. Other investigations of the effect of two-colour fields on the generated high harmonic spectrum have been performed but for very different interaction geometries and parameters to the current work $^{11-12}$.

Here we describe experimental data, supported by numerical simulations, which demonstrate that, ultimately, attosecond control of the relative phase of the two driving fields is required to optimise the XUV enhancement. Our experimental results confirm the theoretical predictions in Edwards et al. ${ }^{10}$, whereas the current simulations extend the parameter range to include values that are relevant to the experiment. The detailed mechanism is first examined in figure 1 using particle-in-cell (PIC) simulations 
for the cases when the relative phase, $\Delta \phi$, of the second harmonic is such that the high harmonic yield is either maximised or minimised. The complete phase dependence is presented later.

First, the case for $\Delta \phi=0$ is examined (figure 1a-1c). The XUV emission of each cycle originates from one primary bunch that is compressed and rapidly accelerated away from the surface to relativistic velocities. This bunch subsequently emits an attosecond pulse before breaking up and returning to the plasma. Some secondary bunches are also generated but do not contribute significantly to the emission for harmonic orders $>20$. This behaviour is a direct result of the formation of a steep gradient with respect to time in the dressed electric field (circled region in Figure 1a). In addition, the bunch is seen to emit an attosecond pulse at the moment when the particle velocities are highest. This maximises the cut-off frequency $\left(\sim \gamma^{3}\right.$ where $\gamma$ is the Lorentz factor $\left.{ }^{9}\right)$ for the generated radiation, which demonstrates that the precisely timed two-colour field matches the XUV emission to the acceleration provided by the fundamental driving laser field.

For $\Delta \phi=\pi$, the formation of a saddle point in the driving field (circled region in figure $1 \mathrm{~d}$ ) staggers the acceleration of the electrons leading to a lower bunch density at the time of emission. Since the emitted power scales with $n_{b}^{2}$ for a bunch with electron density $n_{b}$, this results in weaker XUV radiation in addition to a broad temporal distribution of the emission (figure 1e). Furthermore, the peak velocity of the electrons is significantly smaller than the $\Delta \phi=0$ case and is not synchronised with the time that the bunch emits XUV radiation. These simulations illustrate the degree to which control of the relative phase of the two colours can strongly influence the sub-cycle dynamics of the electron bunches.

Next we investigate if it is possible to achieve this degree of attosecond control in the laboratory as well as the sensitivity of the enhancement to the exact relative phase. Figure 2 shows the setup, consisting of optics for frequency doubling, independent polarisation rotation and timing control, used to collinearly generate the two-colour field. More details can be found in the methods and ref 13. 
Figures 3(a) and (b) show the measured spectral energy density, as the second harmonic phase is varied, for photon energies from $24 \mathrm{eV}$ to $33 \mathrm{eV}$ and from $33 \mathrm{eV}$ to $42 \mathrm{eV}$ respectively. The higher spectral range was chosen for detailed analysis, as it is free of a competing harmonic generation mechanism known as Coherent Wake Emission ${ }^{14}$ (CWE) which can become dominant for lower photon energies. The details of this mechanism and its relation to the current results are discussed in the supplementary information. From the data, a strong phase dependence is observed and control over the harmonic yield is demonstrated, as predicted by simulations. The fast decay of the spectra for higher orders indicates that we are close to the cut-off region ${ }^{2}$. This is apparent in figure 3(c), where the highest order harmonics depend critically on the phase setting. This would suggest that the highest electron velocities (the critical parameter for the cut-off harmonic) are only being reached when the $2^{\text {nd }}$ harmonic phase is such that the harmonic yield is maximised, as suggested in figure $1 \mathrm{c}$.

For the higher harmonic orders the detected signal ranges from near complete suppression to a total measured energy of $125 \pm 20 \mathrm{~nJ}$ across the spectral range for $\Delta \phi=0$. For the full spectrum from $24 \mathrm{eV}$ to $42 \mathrm{eV}$ (16th to 27 th harmonic of the fundamental), an energy of $11.8 \pm 0.7 \mu \mathrm{J}$ is observed at the optimum phase, which is higher than the best optimised HHG sources from the well-established 3-step process in gaseous media ${ }^{15,16}$.

It is well known that the efficiency of relativistic surface harmonic generation is strongly dependent on the scale length of the plasma density gradient ${ }^{17,18}$. Here, the length of the plasma density gradient was controlled by the use of a prepulse mirror (see Methods), as in the work of Kahaly et al. ${ }^{18}$. Our experimental evidence indicates that the harmonic yield using the two-colour field is significantly better when the prepulse is absent (or, equivalently, for the shortest scale length). The total harmonic yield for orders above the $21^{\text {st }}$ was found to be a factor of $57 \pm 10$ higher when the two-colour pulse was used, demonstrating the considerable gains that can be achieved by converting only a small fraction of the 
fundamental laser energy to the second harmonic. More details regarding the effect of the prepulse on the high harmonic yield are given in the supplementary information.

We further investigated the dependence of the harmonic efficiency on the polarisation of the second harmonic (further details can be found in the supplementary information), and observed that the $2^{\text {nd }}$ harmonic only increases the harmonic yield where its polarisation is parallel to that of the fundamental. This is in contrast to two-colour driven HHG in gaseous media, where strong enhancements are also seen for orthogonal polarisation ${ }^{19-21}$.

As this is a collinear setup, the shorter wavelength of the second harmonic implies that this beam is focussed to a smaller spot than that associated with the fundamental (full width at half maximum (FWHM) of $2.7 \mu \mathrm{m}$ for $2 \omega$ vs $4.7 \mu \mathrm{m}$ for $1 \omega)$. We estimate the conversion efficiency relative to the energy of both beams concentrated within the $2 \omega$ spot to be $\approx 1 \times 10^{-3}$ for the full photon energy range from 24 to $42 \mathrm{eV}$, or $\approx 2 \times 10^{-4}$ absolute efficiency considering the full input energy. As the significantly larger spot area of the fundamental still contributes to the recorded spectrum, the spectrum measured without the $2^{\text {nd }}$ harmonic pulse has been subtracted to obtain these numbers so that we only consider the efficiency from the two-colour process.

Figure 4 shows the phase dependence for the integrated yield for harmonics above the $21^{\text {st }}$ for both the experimental and simulation data. Here we see remarkable agreement for a 1D code, further supporting that the origin of this effect lies in the temporal variation of the incident field. Additionally, the signal obtained when there is no $2^{\text {nd }}$ harmonic is also plotted here as a baseline, and is seen to be comparable to the weakest signal seen for the two-colour pulse. Data for additional $2^{\text {nd }}$ harmonic energy fractions and scale lengths are provided in the supplementary information. The plotted phase dependence clearly demonstrates that accurate sub-cycle control over the relative timing is critical for optimising the harmonic yield. 
Overall, this work demonstrates sub-laser cycle control of relativistic electron bunch trajectories by tuning of the relative phase of an added second harmonic pulse. The enhancement in the emitted XUV yield for modest laser peak powers (<2TW) brings this relativistic high harmonic source within the reach of an extremely wide range of lasers which can reach these powers at $10 \mathrm{~Hz}$ repetition rates, and is not far from that achievable with current $\mathrm{kHz}$ systems ${ }^{22}$. Although the current work concerns the generation of pulse trains, it could be combined with a suitable gating scheme $e^{23}$ to isolate single pulses suitable for attosecond science. Extending this method to even higher power systems (using, for example, annularly split beams) has the potential to produce attosecond pulses with unprecedented energies. Using the same intensity with a PW class laser would result in $\mathrm{mJ}$ of harmonic energy in the current spectral range, whilst scaling the intensity to the limits possible with such laser powers is expected to result in substantial increases in yield at higher photon energies. These possibilities require experimental investigation, due to the challenge of performing fully predictive simulations of dense relativistic plasma interactions.

\section{METHODS}

Two-colour field setup

The laser used for this study was the JETI 40 Ti:Sapphire system after passing through a $23 \mathrm{mmX} 23 \mathrm{~mm}$ aperture to ensure the beam size matched the calcite crystal size. This beam was contrast enhanced using a plasma mirror system $^{25}$ in order to ensure a very steep density profile that could be controlled via a translatable prepulse mirror ${ }^{18}$. Without conversion to its second harmonic, this setup yielded pulses of $60 \mathrm{~mJ}$ with $35 \mathrm{fs}$ pulse duration and an intensity of $\approx 3.6 \times 10^{18} \mathrm{Wcm}^{-2}$ on target after focusing by a $15 \mathrm{~cm}$ focal length silver coated off-axis parabola. A $700 \mu \mathrm{m}$ thick potassium dihydrogen phosphate (KDP) crystal was used to frequency double the pulse with $\approx 10 \%$ efficiency yielding an intensity of $\approx 1.1 \times 10^{18} \mathrm{Wcm}^{-2}$ from the second harmonic and a small drop in intensity for the fundamental due to the energy lost from the conversion. The broadening of the $2^{\text {nd }}$ harmonic pulse was estimated to be $\approx 7 \mathrm{fs}$ by numerically modelling the propagation of both pulses through the KDP. The additional $2^{\text {nd }}$ harmonic 
energy added at each time step was taken to be proportional to the square of the intensity of the fundamental pulse. It was found that the broadening due to group velocity mismatch in the crystal was largely compensated by pulse shortening due to the nonlinear conversion of the laser pulse. The time delay between the two colours was precompensated by a $615 \mu \mathrm{m}$ calcite crystal before the beam passed through an achromatic waveplate which introduced a retardation of $1.5 \lambda$ for $400 \mathrm{~nm}$ and $1 \lambda$ for $800 \mathrm{~nm}$ in order to ensure both pulses were P-polarised on target. The total thickness of the waveplate was approximately $1 \mathrm{~mm}$. Finally, the relative phase was tuned by controlling the rotation of a $500 \mu \mathrm{m}$ fused silica wafer thus changing the relative path difference. Temporal overlap was confirmed by optimising third harmonic generation in a BBO crystal.

\section{Spectrometer}

The reflected radiation from the interaction is first filtered by a $200 \mathrm{~nm}$ aluminium foil before it is focused by a $\mathrm{Ni}$ coated toroidal mirror which makes a one-to-one image of the source. This image is spectrally dispersed by a 1000 lines/mm freestanding gold transmission grating and the resulting spectrum is detected on an ANDOR Newton D0940P-BN back illuminated CCD. The spectrometer has an 8mradX6mrad collection angle which, assuming diffraction limited harmonic emission, would be expected to collect almost the entire harmonic beam. This is only valid for the relativistic harmonics if negligible denting of the target ${ }^{26-28}$ is assumed. Without this assumption, the measured values constitute a lower limit for the actual generation efficiency. This spectrometer was previously calibrated at a synchrotron source allowing absolute energy values to be determined ${ }^{29}$.

\section{Scale Length Control}

The plasma surface density gradient was controlled by the use of a $3 \mathrm{~mm}$ thick fused silica substrate with an antireflection coating on the front side and a high reflectivity coating on the reverse. The optic is placed directly in front of one of the full beam mirrors and reflects a small section of the beam which is then focused by the same focusing optic as the main pulse. This lower intensity spot acts as a prepulse, ionising the surface of the target making an expanding plasma. Thus, by controlling the timing of the arrival of the prepulse by translation of this special optic, the length of the expanding plasma at the time the driving pulse arrives can be controlled. The high reflectivity coating of the prepulse mirror is placed on the side closest to the full beam mirror so that the prepulse must propagate through the $3 \mathrm{~mm}$ substrate. The additional delay this introduces means that the prepulse can be 
initially synchronised with the main pulse while maintaining a small gap between the two mirror surfaces, thus permitting a full timing scan to be performed ${ }^{18}$.

PIC Simulations

1D PIC simulations were performed using the code $\mathrm{PICWIG}^{30}$. For the simulations of the phase dependence of the harmonic yield, the cell width used was $\lambda / 500$ ( $\lambda=$ =laser wavelength) and the target had a $\lambda / 50$ scale length modelled as an exponential density ramp at the front of the target leading up to a peak density of $400 n_{c}$ where $n_{c}=\omega^{2} m_{e} \varepsilon_{0} / e^{2}$ is the critical density for an electromagnetic wave with angular frequency $\omega$ and with $m_{e}, \varepsilon_{0}$ and $e$ equal to the electron mass, electric permittivity and electron charge respectively. Ions were mobile and each target cell contained 1000 electron and 1000 ion macroparticles. The laser pulse was modelled by a $\sin ^{2}$ field envelope with a total width of 36.8 laser cycles and peak $a_{0}$ values of 1.25 for the fundamental and 0.36 for the $2^{\text {nd }}$ harmonic. For the simulations yielding the electron density and trajectory data, a shorter pulse (10 cycles) and a cell width of $\lambda / 1000$ was used for improved spatial resolution and the number of particles per cell was reduced to 500 for each species to manage the higher computational load.

\section{REFERENCES}

1. Macchi, A., Borghesi, M. and Passoni, M. Ion acceleration by superintense laser-plasma interaction. Rev. Mod. Phys. 85, 751 (2013)

2. an der Brügge, D. \& Pukhov, A. Enhanced relativistic harmonics by electron nanobunching. Phys. Plasmas $17,033110(2010)$

3. Dromey, B. et al. Coherent synchrotron emission from electron nanobunches formed in relativistic laserplasma interactions. Nature Phys. 8, 804 (2012)

4. Fletcher, L.B. et al. Ultrabright X-ray laser scattering for dynamic warm dense matter physics. Nature Photon. 9, 274 (2015)

5. Krausz, F. and Ivanov, M. Attosecond physics. Rev. Mod. Phys. 81, 163 (2009) 
6. Sansone, G., Poletto, L and Nisoli, M. High-energy attosecond light sources. Nature Photon. 5, 655 (2011)

7. Bulanov, S., Naumova, N.M. \& Pegoraro, F. Interaction of an ultrashort, relativistically strong laser pulse with an overdense plasma. Phys. Plasmas 1, 745 (1994)

8. Lichters, R., Meyer-ter-Vehn, J. \& Pukhov, A. Short-pulse laser harmonics from oscillating plasma surfaces driven at relativistic intensity. Phys. Plasmas 3, 3425-3437 (1996)

9. Baeva, T., Gordienko, S. and Pukhov, A. Theory of high-order harmonic generation in relativistic laser interaction with overdense plasma. Phys. Rev. E 74, 046404 (2006)

10. Edwards, M.R., Platonenko, V. T. \& Mikhailova, J. M. Enhanced attosecond bursts of relativistic high-order harmonics driven by two-color fields. Opt. Lett. 39, 6823 (2014)

11. Mirzanejad, S. \& Salehi, M. Two-color high-order-harmonic generation: Relativistic mirror effects and attosecond pulses. Phys. Rev. A 87, 063815 (2013)

12. Tarasevitch, A. \& von der Linde, D. High order harmonic generation from solid targets: Towards intense attosecond pulses. Eur. Phys. J. Special Topics 175, 35-41 (2009)

13. Dudovich, N. et al. Measuring and controlling the birth of attosecond XUV pulses. Nature Phys. 2, 781 (2006)

14. Quéré, F. et al. Coherent Wake Emission of High-Order Harmonics from Overdense Plasmas. Phys. Rev. Lett. 96, 125004 (2006)

15. Corkum, P.B. Plasma Perspective on Strong-Field Multiphoton Ionization. Phys. Rev. Lett. 71, 1994-1997 (1993)

16. Takahashi, E.J., Lan, P., Mücke, O.D., Nabekawa, Y. \& Midorikawa, K. Nonlinear Attosecond Metrology by Intense Isolated Attosecond Pulses. IEEE J. Sel. Top. Quantum Electron. 21, 8800112 (2015)

17. Zepf, M. et al. Role of the plasma scale length in the harmonic generation from solid targets. Phys. Rev. $E$ 58, R5253 (1998) 
18. Kahaly, S. et al. Direct Observation of Density-Gradient Effects in Harmonic Generation from Plasma Mirrors. Phys. Rev. Lett. 110, 175001 (2013)

19. Kim, I.J. et al. Highly Efficient High-Harmonic Generation in an Orthogonally Polarized Two-Color Laser Field. Phys. Rev. Lett. 94, 243901 (2005)

20. Brugnera, L. et al. Trajectory Selection in High Harmonic Generation by Controlling the Phase between Orthogonal Two-Color Fields. Phys. Rev. Lett. 107, 153902 (2011)

21. Lambert, G. et al. Aberration-free high-harmonic source generated with a two-colour field. EPL 89, 24001 (2010)

22. Zhou, C. et al. Large-Scale, High-Efficiency Transmission Grating for Terawatt-Class Ti:Sapphire Lasers at 1kHz. Appl. Phys. Express 4, 072701 (2011)

23. Yeung, M. et al. Noncollinear Polarization Gating of Attosecond Pulse Trains in the Relativistic Regime. Phys. Rev. Lett. 115, 193903 (2015)

24. Rödel, C. et al. Harmonic Generation from Relativistic Plasma Surfaces in Ultrasteep Plasma Density Gradients Phys. Rev. Lett. 109, 125002 (2012)

25. Rödel, C. et al. High repetition rate plasma mirror for temporal contrast enhancement of terawatt femtosecond laser pulses by three orders of magnitude. Appl. Phys. B 103, 295-302 (2011)

26. Vincenti, H. et al. Optical properties of relativistic plasma mirrors. Nat. Commun. 5, 3403 (2014)

27. Yeung, M. et al. Beaming of High-Order Harmonics Generated from Laser-Plasma Interactions. Phys. Rev. Lett. 110, 165002 (2013)

28. Dromey, B. et al. Diffraction-limited performance and focusing of high harmonics from relativistic plasmas. Nature Phys. 5, 146 (2009)

29. Fuchs, S. et al. Sensitivity calibration of an imaging extreme ultraviolet spectrometer-detector system for determining the efficiency of broadband extreme ultraviolet sources. Rev. Sci. Instrum. 84, 023101 (2013) 
30. Rykovanov, S.G., Geissler, M., Meyer-ter-Vehn, J. \& Tsakiris, G.D. Intense single attosecond pulses from surface harmonics using the polarization gating technique. New J. Phys. 10, 025025 (2008).

\section{ACKNOWLEDGEMENTS}

This work was supported by the European Regional Development Fund (EFRE), Deutsche Forschungsgemeinschaft (SFB TR18-A7), UK Engineering and Physical Sciences Research Council (EPSRC) grant EP/I029206/1 and by Laserlab Europe. This project has received funding from the European Union's Horizon 2020 research and innovation programme under the Marie Sklodowska-Curie grant agreement No. 641272. The authors gratefully acknowledge the computing time granted by the John von Neumann Institute for Computing (NIC) and provided on the supercomputer JURECA at Jülich Supercomputing Centre B.D. acknowledges support from the EPSRC through grants and a Career Acceleration Fellowship. S.R. acknowledges the support by the Helmholtz Association (Young Investigator's Group VH-NG-1037). C.R. acknowledges support from the VolkswagenStiftung. The authors acknowledge the support of Falk Ronneberger and Burgard Beleites as laser operators.

\section{AUTHOR CONTRIBUTIONS}

All authors contributed to the planning and implementation of the experiment. M.Y., S.R. and M.Z. carried out the data analysis and the PIC simulations were performed by S.R.

\section{COMPETING FINANCIAL INTERESTS}

The authors declare no competing financial interests.

Correspondance and requests for materials should be addressed to M.Y. 
Figure 1

Simulations and mechanism for two-colour field driven attosecond pulse emission - (a) Plot of the electric fields of the two-colour pulse (solid blue) and fundamental only pulse (dashed red) for the case with maximum high harmonic yield. $T_{L}$ is one period of the fundamental frequency. (b) Plots against time, $t$, and position, $x$ for the electron density, $n_{e}$, in units of critical density, $n_{c}$, and normalised transverse electric field, $E_{y}$, filtered to remove frequencies below the $21^{\text {st }}$ harmonic. $\lambda_{L}$ is the fundamental wavelength. Magnified region shows the 1. Formation, 2. Acceleration and 3. Emission stages of the first bunch as indicated by the numbered circles. (c) Velocity, $v$, in units of c, plotted against the same time axis as panel $b$ for each of a subset of individual particles. The dotted line indicates the time at which the first bunch reaches its peak velocity. (d-f) Plots are as (a-c) respectively but for the case when the harmonic yield is minimised.

\section{Figure 2}

Experimental setup - A schematic of the optical setup for generating the two-colour field and the spectrometer layout. The timing and polarisation of each frequency is indicated after each stage. The $p$ polarised fundamental is frequency doubled in a potassium dihydrogen phosphate (KDP) crystal, after which the orthogonally polarised second harmonic passes through a calcite time delay compensator so that both the fundamental and second harmonic are temporally overlapped at the target. An achromatic waveplate allows independent control of the $2^{\text {nd }}$ harmonic polarisation before the beam passes through a fused silica wafer which can be tilted in order to control the relative phase between the two frequencies. After focusing to high intensity on target by an off-axis parabola (OAP), the reflected radiation is filtered by an thin aluminium (Al) filter and collected by a toroidal mirror which, after spectral dispersion by a gold transmission grating, is imaged onto an XUV CCD. 
Figure 3

Phase dependence of harmonic spectra - The measured calibrated spectra, each averaged over 10 shots, are plotted here for each relative phase setting for a peak normalised vector potential $\mathrm{a}_{0} \approx 1.25$ (where $\mathrm{a}_{0}{ }^{2}=\mid \lambda^{2} / 1.38 \times 10^{18} \mathrm{Wcm}^{-2} \mu \mathrm{m}^{2}$ for an intensity I and wavelength $\left.\lambda\right)$ for the fundamental beam and peak $\mathrm{a}_{0} \approx 0.36$ for the $2^{\text {nd }}$ harmonic. The spectra for harmonics 16 to 21 are shown in (a) whilst (b) shows spectra for harmonics 22 to 27 . The zero phase position is determined from comparisons with simulations. (c) A false colour map where each row shows the average spectrum for one phase setting of the $2^{\text {nd }}$ harmonic and where each spectrum has been normalised to the peak signal of the complete phase scan and scaled to make the higher orders visible to clearly show the full spectral dependence on the relative phase.

Figure 4

Comparison with simulation -The normalised harmonic yield, integrated over harmonics 22 to 27 (blue crosses) is plotted against the relative phase alongside results from 1D PIC simulations (red circles). The phases are absolute and taken from the simulation parameters while the absolute phase for the experimental data has been set so that the phase for the maximum harmonic yield matches that of the simulations. The shaded region indicates the standard deviation of the experimental data. The lower shaded orange region shows the same variation of the signal in this spectral range for the case when there is no $2^{\text {nd }}$ harmonic present and the prepulse position has been optimised. The red dashed line indicates the simulation result for the optimal scale length for the fundamental only for an equivalent comparison. Note that the discrepancy in the absolute enhancement with the simulation can be attributed to the $1 \mathrm{D}$ simulations not accounting for variation of the $2^{\text {nd }}$ harmonic intensity ratio across the focal region as well as differences in the absolute efficiencies observed due to spatial effects that are not considered ${ }^{24}$. 


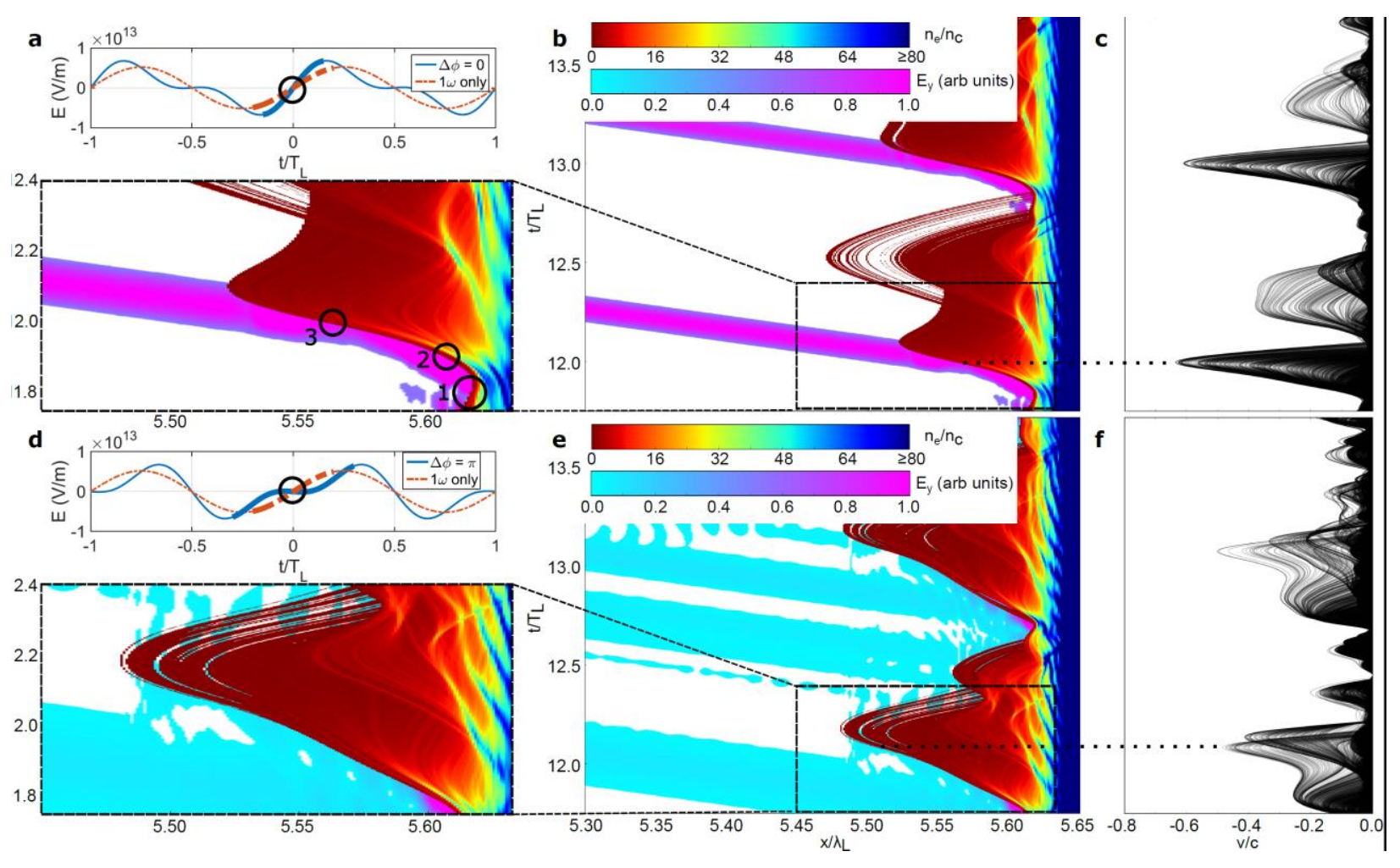

Figure 1

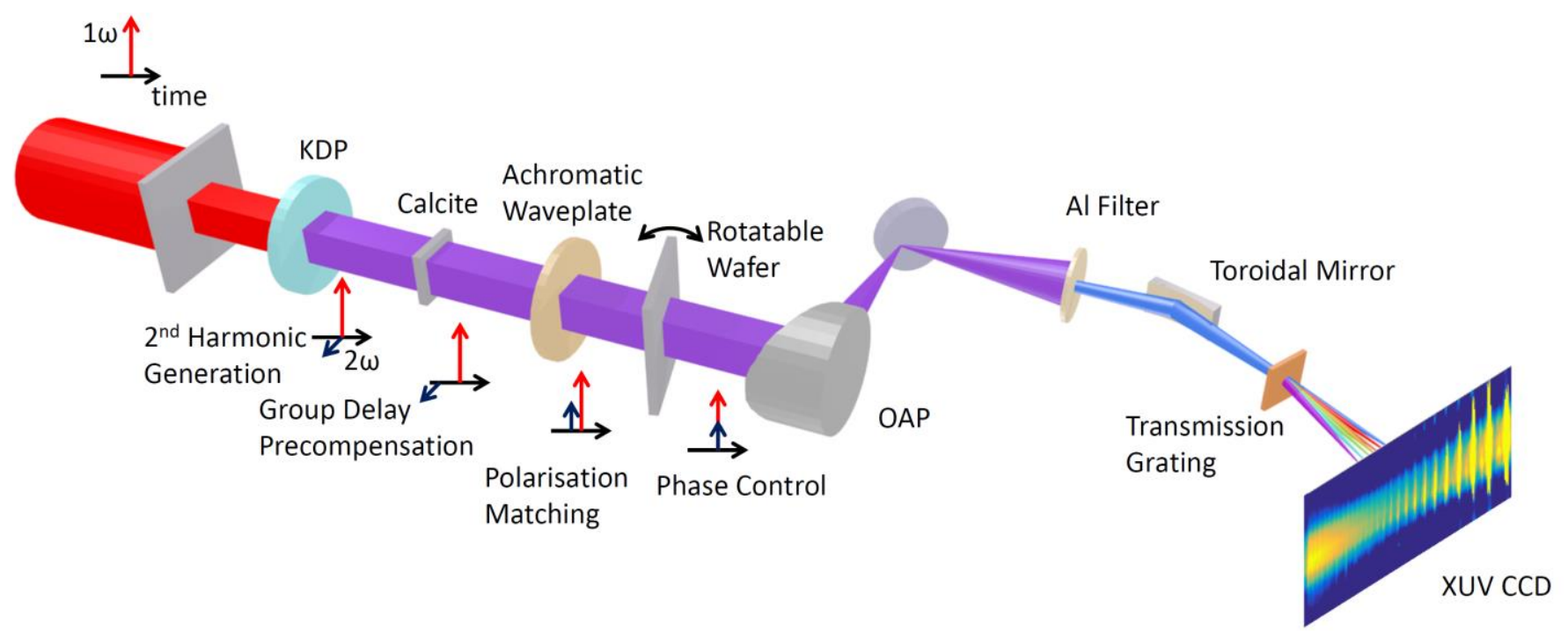

Figure 2 

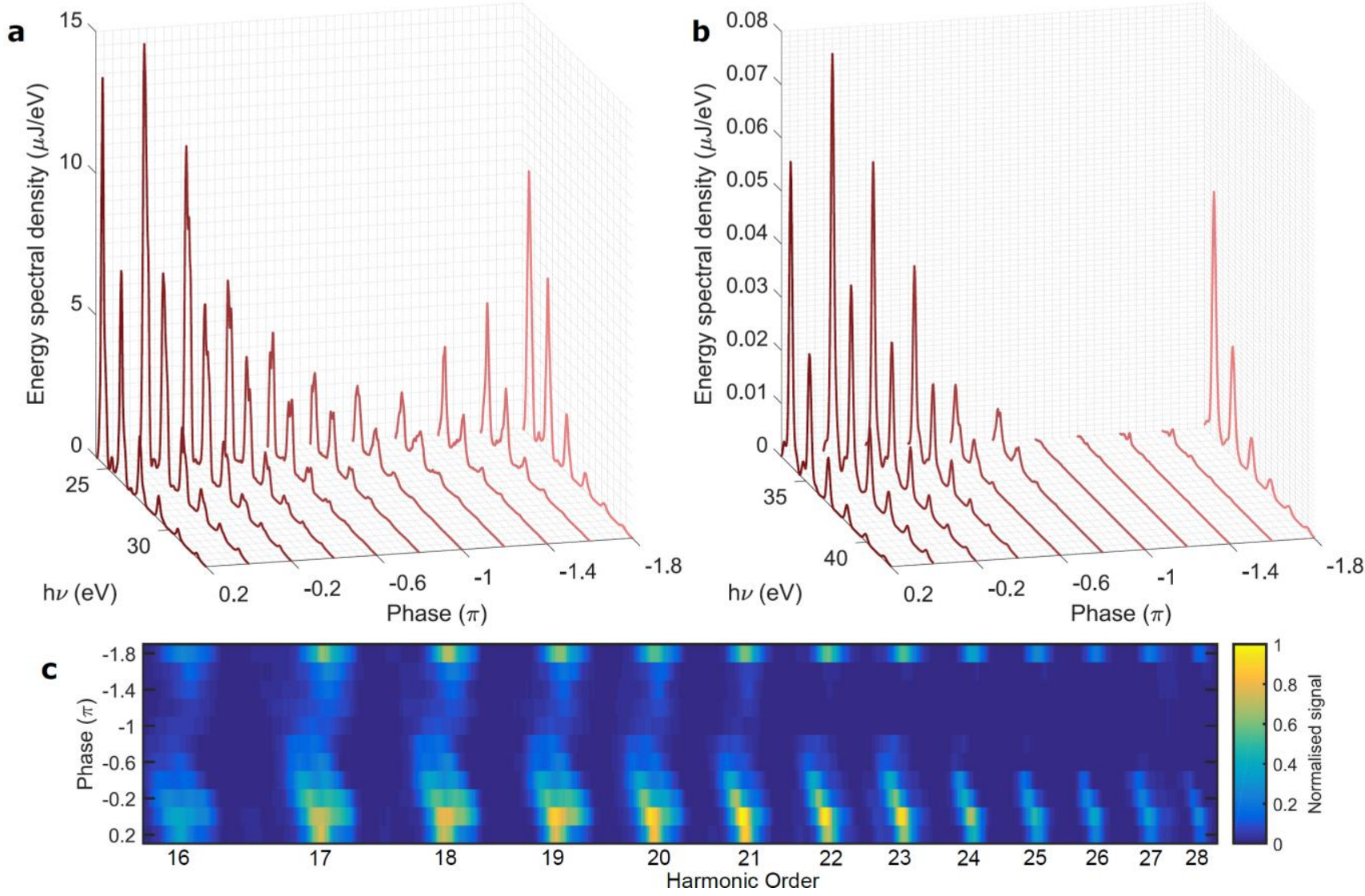

Figure 3

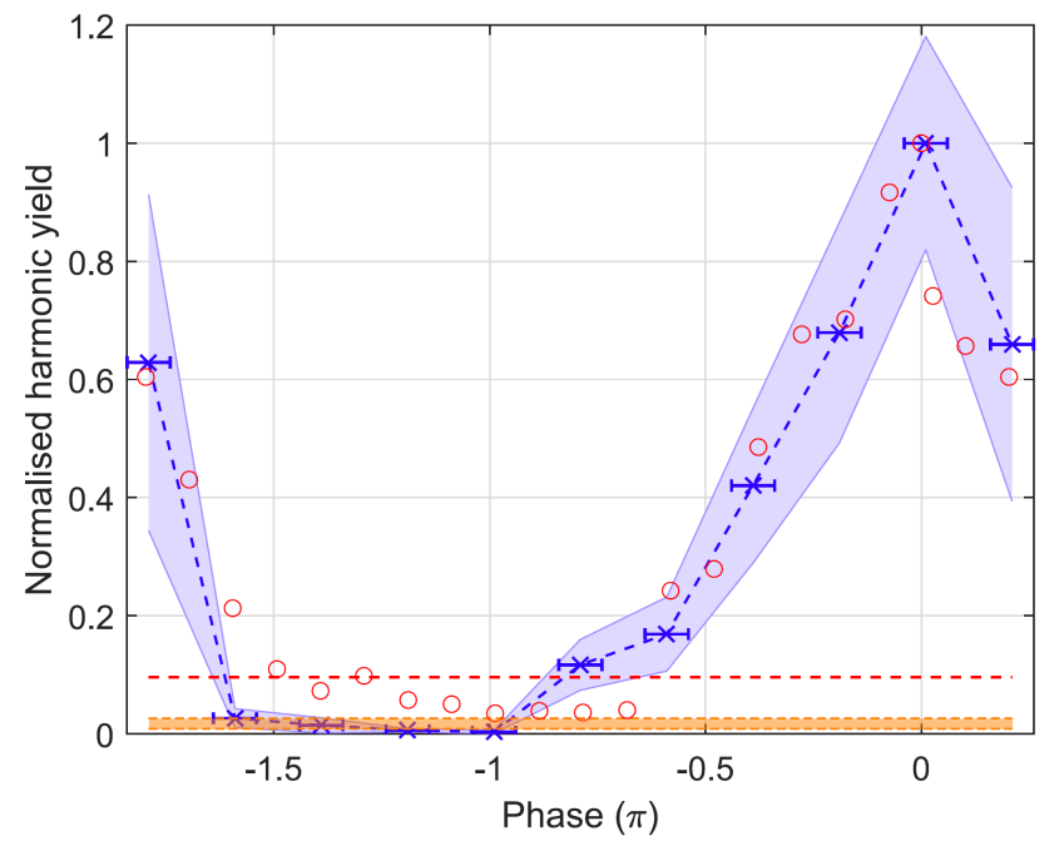

Figure 4 